\title{
Compressive Sensing Based Channel Estimation for High-order MIMO Systems
}

\author{
https://doi.org/10.3991/ijoe.v14i07.8218 \\ Zhiguo Lv \\ Xidian University, Xi'an, China \\ Luoyang Institute of Science and Technology, Luoyang, China \\ Ying $\operatorname{Li}^{(\varpi)}$ \\ Xidian University, Xi'an, China \\ yli@mail.xidian.edu.cn
}

\begin{abstract}
The high-order multiple-input multiple-output (MIMO) system can remarkably increase the data rate or enhance the reliability. However, it is difficult to perform channel estimation because of the massive number of antennas. The Narrow Band Estimation Antenna Processing (NBEAP) scheme is used to deal with this issue. Nevertheless, the accuracy of the channel estimation needs to be improved. In this paper, a compressive sensing based scheme named Narrow Band Estimation Fixed Antenna Processing (NBEFAP) is proposed to estimate the channel state information (CSI) for high-order MIMO systems. A simple pilot structure is designed to decrease the computation complexity. In addition, the pilot length is adjusted according to the time-varying sparsity level of the CSI. Compared with NBEAP scheme, NBEFAP scheme can improve the estimation error performance. Simulation results verify the effectiveness of the NBEFAP scheme.
\end{abstract}

Keywords-Channel estimation, high-order MIMO, compressive sensing, CSI

\section{Introduction}

High-order multiple-input multiple-output (MIMO) system can bring great improvements on channel capacity and spectral efficiency [1-3]. However, the estimation of channel state information (CSI) becomes a challenging problem due to the massive number of antennas. In the conventional channel estimation scheme, the receiver estimates the channel depending on the received pilot sequences sent from different transmitting antennas. Generally, any two pilot sequences from different antennas should be mutually orthogonal to enable the identification of the target transmitting antennas. In order to guarantee the orthogonality, the length of pilot sequences should be greater than the number of transmitting antennas. As a result, the length of the pilot sequence is very large in high-order MIMO system. Nevertheless, the total length of the pilot data and information data is limited by the coherence time of the channel. Therefore, a long pilot sequence would result in low information 
transmission efficiency. These observations motivate us to design a scheme which can estimate the CSI with shorter pilot sequences.

Recently, the compressive sensing (CS) [4-7] technique has been applied to reconstruct the sparse signal. In the CS theory, the high dimension sparse signal can be reconstructed by the low dimension measurement signal with high accuracy. On the other hand, recent experiments have shown that many wireless channels are either sparse or approximate sparse [8-10]. For some wireless channels which are not sparse, they can be represented as sparse virtual channels [11][12]. Therefore, the CS technique exhibits great potentials in the field of channel estimation.

A channel feedback reduction techniques based on CS was proposed in [13]. However, the attention was only paid to the CSI feedback from the receivers to the transmitters, rather than the estimation of the CSI. A low-rank matrix approximation based on CS was proposed to estimate the channel and solved via a quadratic semi-define programming in [14]. However, the estimation error performance of this scheme was not considered when the pilot sequence length is shorter than the number of the transmitting antennas. An adaptive one-bit compressed sensing scheme for channel estimation was proposed in [15], where the precoding and combining vectors are designed to increase the accuracy of the channel estimation depending on one-bit feedback from the receiver. Although the estimation accuracy is improved, the feedback mechanism would result in reductions of the information transmission efficiency. A CS based channel estimation scheme was proposed in [16], where the CSI at the desired angle of arrival (AoA) and angle of departure (AoD) is obtained by selecting appropriate precoding and combining vectors. However, neither of the schemes in [15] and [16] has a detailed analysis of the measurement matrix which has a great influence on the channel estimation accuracy.

Bajwa, Sayeed and Nowak proposed another CS based scheme named narrow band estimation-antenna processing (NBEAP) [17]. In NBEAP scheme, a portion of transmitting antennas were chosen randomly to transmit the pilot signal, while the others keep silent. The estimation for the virtual sparse channel is performed by processing the pilot signals received on the randomly selected receiving antennas. However, the received signals are not fully exploited by this scheme. Besides, it is inconsistent with the actual situation to assume the channel sparsity level is priori known and timeinvariant.

In this paper, a modified scheme named Narrow Band Estimation Fixed Antenna Processing (NBEFAP) is proposed. In the NBEFAP scheme, a simple pilot structure is designed to reduce the computational complexity. Moreover, the length of pilot sequence is adjusted flexibly according to the sparsity level of the channel. Simulation results verify that the NBEFAP scheme could guarantee the stability of the estimation error performance at different channel sparsity levels.

Notations: We use lower-case (upper-case) bold characters to denote vectors (matrixes). $\mathbb{N}\left(0, \sigma^{2}\right)$ denotes a Gaussian distribution with mean 0 and variance $\sigma^{2} . \mathbf{I}_{N}$ denotes an $N \times N$ identity matrix. In addition, $(\cdot)^{T},(\cdot)^{H}$ and $\otimes$, denote transpose, Hermitian transpose and Kronecker product respectively. The Frobenius norm, $\ell_{0}$ and $\ell_{2}$ vector norm are denoted as $\|\cdot\|_{F},\|\cdot\|_{\ell_{0}}$ and $\|\cdot\|_{\ell_{2}}$, respectively. We also denote by $\operatorname{vec}\left(\right.$.) the column vector obtained by concatenating the columns of a matrix, $[.]_{i, j}$ 
the $(i, j)$ th entry of the matrix, $[.]_{\mathrm{L}}$ the first $L$ columns of a matrix and $[.]_{\mathrm{Lth}}$ the $L$ th column of a matrix.

\section{Channel models}

Consider a high-order MIMO wireless communication system equipped with $P$ transmitting antennas and $Q$ receiving antennas, where $P \gg 1$ and $Q \gg 1$. There are $N_{\text {path }}$ propagation paths in the physical channel. The gain of the $n$th path is denoted by $\beta$. The normalized angle of arrival and departure at the nth path are $\theta_{R, n}$ and $\theta_{T, n}$ , respectively. They are given by

$$
\begin{aligned}
& \theta_{R, n}=\frac{d \sin \left(\phi_{R, n}\right)}{\lambda} \\
& \theta_{T, n}=\frac{d \sin \left(\phi_{T, n}\right)}{\lambda},
\end{aligned}
$$

where $\lambda$ is the wavelength of propagation, $d$ is the antenna spacing, $\phi_{R, n}$ and $\phi_{T, n}$ are physical AoA and AoD of the $n$th path, respectively. The corresponding response and steering vectors are $\boldsymbol{\alpha}_{R}\left(\theta_{R, n}\right)$ and $\boldsymbol{\alpha}_{T}\left(\theta_{T, n}\right)$, respectively. They are given by

$$
\begin{aligned}
& \boldsymbol{\alpha}_{R}\left(\theta_{R, n}\right)=\frac{1}{\sqrt{Q}}\left[1, e^{-j 2 \pi \theta_{R, n}}, \cdots, e^{-j 2 \pi(Q-1) \theta_{R, n}}\right]^{T} \\
& \boldsymbol{\alpha}_{T}\left(\theta_{T, n}\right)=\frac{1}{\sqrt{P}}\left[1, e^{-j 2 \pi \theta_{T, n}}, \cdots, e^{-j 2 \pi(P-1) \theta_{T, n}}\right]^{T} .
\end{aligned}
$$

As a result, the physical MIMO channel is modeled as

$$
\mathbf{H}=\sum_{n=1}^{N_{\text {path }}} \beta_{n} \boldsymbol{\alpha}_{R}\left(\theta_{R, n}\right) \boldsymbol{\alpha}_{T}{ }^{H}\left(\theta_{T, n}\right) .
$$

Generally, we uniformly quantify the physical propagation path into $P$ virtual AoD and $Q$ virtual AoA. The $p$ th virtual AoD and $q$ th virtual AoA correspond to a virtual direction matrix and a virtual path. The virtual direction matrix is given by

$$
\mathbf{A}_{q, p}=\boldsymbol{\alpha}_{R}\left(\theta_{R, n}\right) \boldsymbol{\alpha}_{T}^{H}\left(\theta_{T, n}\right) .
$$

The gain of the virtual path is the superposition of the physical path gain around it. It is given by

$$
\left[\mathbf{H}_{v}\right]_{q, p}=\sum_{n \in s_{R, q} \cap s_{T, p}} \beta_{n} .
$$

$\left[\mathbf{H}_{v}\right]_{q, p}$ is the virtual path gain related to the $q$ th virtual AoA and $p$ th virtual AoD. $s_{R, q}\left(\right.$ resp. $s_{T, p}$ ) denotes the set of paths whose physical AoA (resp. AoD) lies within the resolution bin centered around the $q$ th (resp. $p$ th) normalized virtual receive (resp. departure) angle $\frac{2 d p}{\lambda P}$ (resp. $\frac{2 d q}{\lambda Q}$ ). They are given by

$$
s_{T, p}=\left\{n: \theta_{T, n} \in\left(\frac{2 d p}{\lambda P}-\frac{d}{\lambda P}, \frac{2 d p}{\lambda P}+\frac{d}{\lambda P}\right)\right\}
$$




$$
S_{R, q}=\left\{n: \theta_{R, n} \in\left(\frac{2 d q}{\lambda Q}-\frac{d}{\lambda Q}, \frac{2 d q}{\lambda Q}+\frac{d}{\lambda Q}\right)\right\} .
$$

Then, the physical channel matrix in (5) is calculated by summing all virtual direction matrices multiplied by corresponding virtual path gain. It is written by

$$
\mathbf{H}=\frac{1}{\sqrt{P Q}} \sum_{q=-\widetilde{Q}}^{\widetilde{Q}} \sum_{p=-\tilde{P}}^{\widetilde{P}}\left[\mathbf{H}_{v}\right]_{q, p} \mathbf{A}_{q, p}
$$

where $\tilde{P}=(P-1) / 2$ and $\widetilde{Q}=(Q-1) / 2$. Here, we set $P$ and $Q$ are odd. The representation of channel in (10) is decoupled as

$$
\mathbf{H}=\widetilde{\mathbf{A}}_{R} \mathbf{H}_{v} \widetilde{\mathbf{A}}_{T}{ }^{H}
$$

where $\widetilde{\mathbf{A}}_{R}$ and $\widetilde{\mathbf{A}}_{T}$ are discrete Fourier transform matrices which are written as

$$
\begin{aligned}
& \widetilde{\mathbf{A}} R=\left[\boldsymbol{\alpha}_{R}\left(-\frac{\widetilde{Q}}{Q}\right), \cdots, \boldsymbol{\alpha}_{R}\left(\frac{\widetilde{Q}}{Q}\right)\right] \\
& \widetilde{\mathbf{A}} T=\left[\boldsymbol{\alpha}_{\mathrm{T}}\left(-\frac{\widetilde{\mathrm{P}}}{\mathrm{P}}\right), \cdots, \boldsymbol{\alpha}_{\mathrm{T}}\left(\frac{\widetilde{\mathrm{P}}}{\mathrm{P}}\right)\right] .
\end{aligned}
$$

Each entry in $\mathbf{H}_{v}$ represents the gain of the channel corresponding to a virtual path. The value of zero means that there is no propagation path between a pair of virtual AoA and AoD. When the number of nonzero entries in the matrix $\mathbf{H}_{v}$ is very small compared to the total number of entries in $\mathbf{H}_{v}$, we call $\mathbf{H}_{v}$ is sparse. The number of the nonzero entries in $\mathbf{H}_{v}$ is defined as the sparsity level of the channel which is denoted by $k$.

Based on the sparse virtual channel model, the task of channel estimation is performed by estimating the sparse matrix $\mathbf{H}_{v}$. In next section, we will introduce an NBEFAP scheme to estimate $\mathbf{H}_{v}$.

\section{NBEFAP scheme}

Our scheme mainly focuses on how to make channel estimation of high-order MIMO systems more efficient. The scheme is described below.

\subsection{Pilot structure}

The first $N_{l}$ transmitting antennas are selected to transmit the pilot signal in turn. When one transmitting antenna transmits the pilot signal, the remaining transmitting antennas remain silent. All the pilot signals are the same. For convenience, " 1 " is used to represent the transmit antenna transmits the pilot and " 0 " for silent. Then, the pilot matrix $\boldsymbol{\Phi}$ of size $P \times N_{l}$ is represented as 


$$
\boldsymbol{\Phi}=\left[\begin{array}{ccccc}
1 & 0 & 0 & \cdots & 0 \\
0 & 1 & 0 & \cdots & 0 \\
\vdots & \vdots & \vdots & \cdots & \vdots \\
0 & 0 & 0 & \cdots & 1 \\
0 & 0 & 0 & \cdots & 0 \\
0 & 0 & 0 & \cdots & 0
\end{array}\right] .
$$

Thus, the received pilot matrix $\mathbf{Y}$ is obtained as

$$
\mathbf{Y}=\mathbf{H} \boldsymbol{\Phi}+\mathbf{W}=\widetilde{\mathbf{A}}_{R} \mathbf{H}_{v} \widetilde{\mathbf{A}}_{T}^{H} \boldsymbol{\Phi}+\mathbf{W}=\widetilde{\mathbf{A}}_{R} \mathbf{H}_{v}\left[\widetilde{\mathbf{A}}^{H}\right]_{N_{l}}+\mathbf{W} .
$$

Due to the special pilot structure, the result of multiplication $\widetilde{\mathbf{A}}_{T}^{H} \boldsymbol{\Phi}$ is directly obtained by taking the first $N_{l}$ columns of $\widetilde{\mathbf{A}}_{T}^{H}$. Therefore, the computational complexity is decreased.

\subsection{Processing of the received pilot signal}

Depend on the received pilot signals on all receiving antennas, the virtual channel $\mathbf{H}_{v}$ is recovered. Since $\widetilde{\mathbf{A}}_{R}$ is a unitary matrix, $\widetilde{\mathbf{A}}_{R} \widetilde{\mathbf{A}}_{R}^{H}=\mathbf{I}_{Q}$. Multiplying both sides of equation (15) by matrix $\widetilde{\mathbf{A}}_{R}^{H}$, we obtain

$$
\widetilde{\mathbf{A}}_{R}^{H} \mathbf{Y}=\mathbf{H}_{v}\left[\widetilde{\mathbf{A}}_{T}^{H}\right]_{N_{l}}+\widetilde{\mathbf{A}}_{R}^{H} \mathbf{W} .
$$

Then the channel estimation problem is written as

$$
\widehat{\mathbf{H}}_{v}=\operatorname{argmin}_{\mathbf{H}_{v}}\left\|\operatorname{vec}\left(\mathbf{H}_{v}\right)\right\|_{\ell 0} \quad \text { s.t. } \widetilde{\mathbf{A}}_{R}^{H} \mathrm{Y}=\mathbf{H}_{v}\left[\widetilde{\mathbf{A}}_{T}^{H}\right]_{N_{l}} .
$$

When the $n$th transmitting antenna sends the pilot signal while the others keep silent, the received signal is

$$
[\mathbf{Y}]_{n t h}=\mathbf{H} \boldsymbol{\Phi}_{n t h}+\mathbf{W}_{n t h}=\widetilde{\mathbf{A}}_{R} \mathbf{H}_{v}\left[\widetilde{\mathbf{A}}_{T}^{H}\right]_{n t h}+[\mathbf{W}]_{n t h} .
$$

Picking out the $m$ th entry from $[\mathbf{Y}]_{n t h}$, we have

$$
\begin{aligned}
{[\mathbf{Y}]_{m, n} } & =\left(\left(\widetilde{\mathbf{A}}_{R}^{T}\right)_{m t h}\right)^{T} \mathbf{H}_{v}\left(\widetilde{\mathbf{A}}_{T^{H}}^{H}\right)_{n t h}+[\mathbf{W}]_{m, n} \\
& =\left(\left(\widetilde{\mathbf{A}}^{H}\right)_{n t h}\right)^{T} \otimes\left(\left(\widetilde{\mathbf{A}}^{T}\right)_{m t h}\right)^{T} \operatorname{vec}\left(\mathbf{H}_{v}\right)+[\mathbf{W}]_{m, n} .
\end{aligned}
$$

It is obvious that $[\mathbf{Y}]_{m, n}$ is related with all the entries of $\mathbf{H}_{v}$. Therefore, each entry of $\mathbf{Y}$ should be exploited to reconstruct $\mathbf{H}_{v}$.

When the pilot sequence length $L$ satisfies the restricted condition $L \geq \frac{K}{Q} \log \frac{Q P}{K}$ [18], the virtual channel $\mathbf{H}_{v}$ is reconstructed as

$$
\begin{aligned}
& \widehat{\mathbf{H}}_{v}=\operatorname{argmin}_{\mathbf{H}_{v}}\left\|\operatorname{vec}\left(\mathbf{H}_{v}\right)\right\|_{\ell 0} \quad \text { s.t. } \operatorname{vec}\left([\mathbf{Y}]_{L}\right)=\operatorname{Mvec}\left(\mathbf{H}_{v}\right), \\
& \text { where } \mathbf{M}=\left(\left(\left(\widetilde{\mathbf{A}}_{T}^{H}\right)_{1 s t}\right)^{T} \otimes \widetilde{\mathbf{A}}_{R} ; \ldots ;\left(\left(\widetilde{\mathbf{A}}_{T}^{H}\right)_{L t h}\right)^{T} \otimes \widetilde{\mathbf{A}}_{R}\right) .
\end{aligned}
$$


When the channel sparsity level $k$ satisfies the restricted condition $\frac{K}{Q} \log \frac{Q P}{K} \leq 1$, we reconstruct $\mathbf{H}_{v}$ by one column of $\mathbf{Y}$, i.e., the required pilot sequence length can be as small as 1 . In this case, the received pilot signal is expressed as

$$
[\mathbf{Y}]_{n t h}=\mathbf{\Omega} \operatorname{vec}\left(\mathbf{H}_{v}\right)+[\mathbf{W}]_{n t h},
$$

where $\boldsymbol{\Omega}=\left(\left(\widetilde{\mathbf{A}}_{T}^{H}\right)_{n t h}\right)^{T} \otimes \widetilde{\mathbf{A}}_{R}$. According to equations (17) and (21), the channel estimation problem is simplified as

$$
\widehat{\mathbf{H}}_{v}=\operatorname{argmin}_{\mathbf{H}_{v}}\left\|\operatorname{vec}\left(\mathbf{H}_{v}\right)\right\|_{\ell 0} \quad \text { s.t. }[\mathbf{Y}]_{n t h}=\mathbf{\Omega v e c}\left(\mathbf{H}_{v}\right) .
$$

\subsection{Adjustment of the pilot sequence length}

The sparsity level $k$ is varied with time in practice. Hence, the transmitter adjusts the length of the pilot sequences according to the sparsity level of the channel to improve the transmission efficiency.

The channel estimation is performed once in a certain amount of information and pilot data which is called a data block. The pilot length is $P$ in the first block. After obtaining the estimated virtual channel, the sparsity level and the required pilot length are computed at the receiver. Then, the required pilot length is fed back to the transmitter. In the second and subsequent blocks, the receiver performs the channel estimation and makes comparison between the current channel sparsity level and the previous one. " 0 " is fed back to the transmitters if the sparsity level decreases. Otherwise, " 1 " is fed back. Accordingly, the transmitters would increase or reduce the pilot length by $\Delta L$ with feedback information " 1 " or " 0 ".

The Euclidean distance between the ideal constellation points and the received points increases with sparsity level. So the average Euclidean distance (AED) is used in practice instead of the sparsity level. The AED is written as

$$
d_{A E D}=\frac{\|\hat{\mathbf{H}}(\hat{\mathbf{x}}-\hat{\mathbf{x}})\|_{\ell_{2}}}{\|\hat{\mathbf{H}}\|_{F}}=\frac{\|\hat{\mathbf{H}} \mathbf{x}-\mathbf{Y}\|_{\ell_{2}}}{\|\hat{\mathbf{H}}\|_{F}},
$$

where $\hat{\mathbf{x}}$ denotes the computed constellation vector and $\tilde{\mathbf{X}}$ denotes the ideal constellation vector, $\mathbf{Y}$ denotes the received pilot signal and $\widehat{\mathbf{H}}$ denotes the estimated channel matrix. Since $\widehat{\mathbf{H}}$ and $\widetilde{\mathbf{x}}$ have been calculated during the decoding of the information data. Therefore, the calculation of the AED is easy to perform.

If the AED increases, the pilot sequence length should be increased. Thus, the receiver feeds " 1 " back to the transmitter. Similarly, it would result in feedback " 0 " when the AED is decreased. The transmitter increases or reduces the pilot length by $\Delta L$ when feedback " 1 " or " 0 ". The adjustment interval $\Delta L$ depends on the variation rate of the channel sparsity level, i.e., faster varying channel requires larger $\Delta L$. 


\section{Discussion of the NBEFAP scheme}

In this section, we discuss the advantages of the proposed NBEFAP scheme. The computational complexities of different schemes are compared. The mutual coherence of the measurement matrix is also discussed since it has a great influence on the estimation accuracy.

\subsection{Features of NBEFAP scheme}

There are three features of our proposed NBEFAP scheme which is described below.

1. The fixed first $N_{l}$ antennas are chosen to transmit the pilot signal sequentially in the proposed NBEFAP scheme. This design generates a simple pilot structure which decreases the calculation complexity in equation (15).

2. The received pilot signals on each receiving antenna have been fully utilized in the proposed NBEFAP scheme. This results in an accurate estimated channel.

3. The pilot sequence length is adjusted according to the sparsity level of $\mathbf{H}_{v}$ to save pilot resource. Compared with traditional schemes with fixed pilot sequence length, the proposed NBEFAP scheme improves the transmission efficiency.

\subsection{Complexity analysis}

For a high-order MIMO system equipped with $P$ transmitting antennas and $Q$ receiving antennas, we use a $L$-length pilot sequence to estimate the channel with different schemes.

The computational complexity of the proposed NBEFAP and conventional NBEAP scheme all mainly concentrate on the reconstruction of sparse channel. Sparse channel reconstruction can be implemented by Matching Pursuit (MP) or Orthogonal Matching Pursuit (OMP) algorithm. The iterations is set to $T$ when MP or OMP algorithm is employed. The computational complexity of the least squares (LS) and linear minimum mean square error (LMMSE) algorithm are also given. The complexity comparison with different schemes is given in table 1. It shows that the proposed scheme has a larger complexity. This is a tradeoff between complexity and accuracy.

Table 1. Complexity comparison

\begin{tabular}{|l|l|}
\hline scheme & complexity \\
\hline LS & $\mathcal{O}(P Q L)$ \\
\hline LMMSE & $\mathcal{O}\left(L^{3}\right)$ \\
\hline MP & $\mathcal{O}\left(L P Q^{2} T\right)$ \\
\hline OMP & $\mathcal{O}\left(L P Q^{2} T+L Q T^{3}\right)$ \\
\hline
\end{tabular}




\subsection{Mutual coherence}

The mutual coherence of a matrix $\mathbf{M}$ is defined as the maximum absolute value of the cross-correlations between columns of $\mathbf{M}$. It is represented as

$$
\mu(\mathbf{M})=\max _{1 \leq i \neq j \leq N} \frac{\left|\left([\mathbf{M}]_{i t h}\right)^{H}[\mathbf{M}]_{j t h}\right|}{\left\|[\mathbf{M}]_{i t h}\right\|_{\ell_{2}}\left\|[\mathbf{M}]_{i t h}\right\|_{\ell_{2}}} .
$$

The mutual coherence will take the minimum value of 0 when the columns in $\mathbf{M}$ are mutually orthogonal. When there are two columns that are linearly related, the mutual coherence will take the maximum value of 1 . According to [19], a smaller $\mu(\mathbf{M})$ of the measurement matrix will bring a more accurate recovery of $\operatorname{vec}\left(\mathbf{H}_{v}\right)$. Usually it is required that the measurement matrix satisfies the mutual coherence property (MCP), i.e., $\frac{\mu(\mathrm{M})+1}{4 \mu(\mathrm{M})} \geq k$, here $k$ is the sparsity level. Therefore, we need to analyze how to reduce the mutual coherence of the measurement matrix.

The domain of values of physical AoA is $\left[-\frac{\pi}{2}, \frac{\pi}{2}\right]$. It is symmetric with respect to origin. As a result, the domain of values of virtual AoA is $\left[-\frac{Q-1}{2 Q}, \frac{Q-1}{2 Q}\right]$. It is also symmetric with respect to origin. For $\widetilde{\mathbf{A}}_{R}=\left[\boldsymbol{\alpha}_{R}\left(-\frac{\tilde{Q}}{Q}\right), \cdots, \boldsymbol{\alpha}_{R}\left(\frac{\tilde{Q}}{Q}\right)\right]$, the column vector $\boldsymbol{\alpha}_{R}(\theta)$ is the conjugation of another column $\boldsymbol{\alpha}_{R}(-\theta)$. Besides, the $\frac{Q+1}{2}$ th column in $\widetilde{\mathbf{A}}_{R}$ is $\boldsymbol{\alpha}_{R}(0)$. All the elements of $\boldsymbol{\alpha}_{R}(0)$ are the same. Therefore, the measurement matrix $\mathbf{M}=\left(\left(\left(\widetilde{A}_{T}^{H}\right)_{1 s t}\right)^{T} \otimes \widetilde{A}_{R} ; \ldots ;\left(\left(\widetilde{A}_{T}^{H}\right)_{L t h}\right)^{T} \otimes \widetilde{A}_{R}\right)$ has two columns that are linearly related. The mutual coherence will take the maximum value, i.e., $\mu(\mathbf{M})=1$. As a conclusion, the domain of AoA cannot be set to symmetry.

When the first column of $\boldsymbol{\Phi}$ is used alone as a pilot, the measurement matrix is written as $\mathbf{M}=\left(\left(\left(\widetilde{\mathbf{A}}_{T}^{H}\right)_{1 s t}\right)^{T} \otimes \widetilde{\mathbf{A}}_{R}\right)$. Since all the elements in $\left[\widetilde{\mathbf{A}}_{T}^{H}\right]_{1 s t}$ are the same, M will contain $Q$ identical columns. As a result, $\mu(\mathbf{M})=1$. So the first column of $\boldsymbol{\Phi}$ must not be used alone as a pilot.

Regardless of the pilot sequence length, the number of columns in $\mathbf{M}$ is $Q P$. Increasing the pilot length would only lead to a larger number of rows in $\mathbf{M}$ as well as a smaller cross-correlation between columns of $\mathbf{M}$. Therefore, a long pilot sequence brings a small mutual coherence.

\section{$5 \quad$ Simulation results}

In this section, we give some simulation results. All the simulations are carried out on Matlab platform. Firstly, a virtual sparse channel model is set up on Matlab platform. Then, a measurement matrix is obtained by setting appropriate channel model parameters. Finally, the OMP reconstruction algorithm is used to estimate the virtual sparse channel. Through the simulation experiment, the influence of channel 
model parameters such as pilot sequence length, channel sparse levels and the number of antennas on channel estimation performance is given.

The accuracy of channel estimation is evaluated by the normalized mean squared error (MSE). It is calculated by

$$
E_{\mathrm{MSE}}=10 \lg \left(\frac{\|\mathbf{H}-\mathbf{H}\|_{F}^{2}}{Q P}\right),
$$

where $\widehat{\mathbf{H}}$ and $\mathbf{H}$ are the estimated and actual channel matrix, $Q$ and $P$ represent the number of receiver and transmitter antennas, respectively. If no special statement, the model parameters used in this paper are listed as follows: transmitter antenna $P=41$, receiver antenna $Q=61$, AoA $\in\left[-\frac{\pi}{2}, \frac{19 \pi}{40}\right]$, AoD $\in\left[-\frac{\pi}{2}, \frac{\pi}{2}\right]$, sparsity level $k=20$ and antenna spacing $d=0.5 \lambda$.

The MSE performance of the channel estimation with different columns of identity matrix as pilot is given in Fig. 1. We can see that using the first column of identity matrix as pilot leads to worse MSE performance than other columns at high Signal Noise Ratio (SNR). This is due to the fact that using the first column of identity matrix alone as pilot decreases the probability of measurement matrix satisfying the MCP.

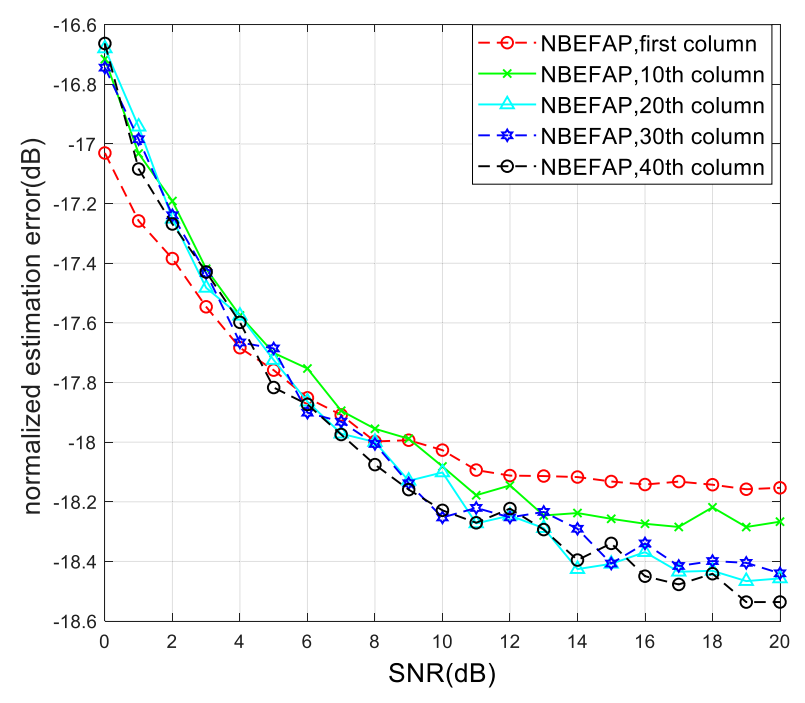

Fig. 1. MSE performance of estimation with different pilot, $k=20, L=1$

The estimation MSE performance of NBEFAP scheme with different pilot sequence lengths is shown in Fig. 2. It is shown that the channel estimation MSE performance is improved with the increase of the pilot sequence length. The reason is that a long pilot sequence provides a small mutual coherence, which improves the accuracy of the channel estimation. 
The estimation MSE performance of NBEFAP scheme with different channel sparsity levels is given in Fig. 3. The simulation result shows that the estimation MSE performance is better when the sparsity level is small. When SNR $=10 \mathrm{~dB}$, the MSE is about $-52 \mathrm{~dB}$ in case of $k=5$. Compared with the cases of $k=10$ and 20 , there are MSE performance gains of $15 \mathrm{~dB}$ and $25 \mathrm{~dB}$, respectively.

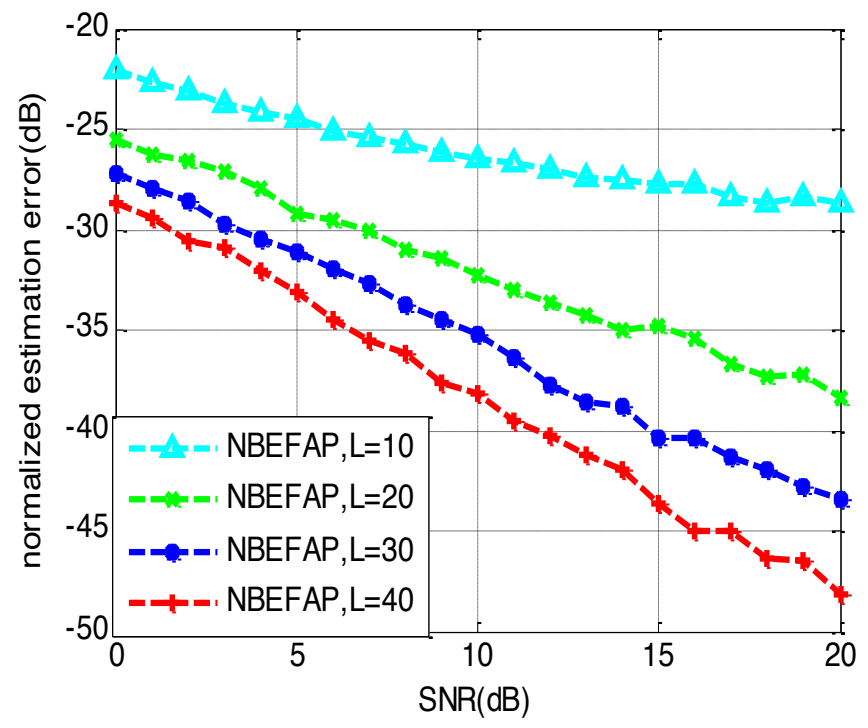

Fig. 2. MSE performance versus SNR with different pilot length, $k=20$

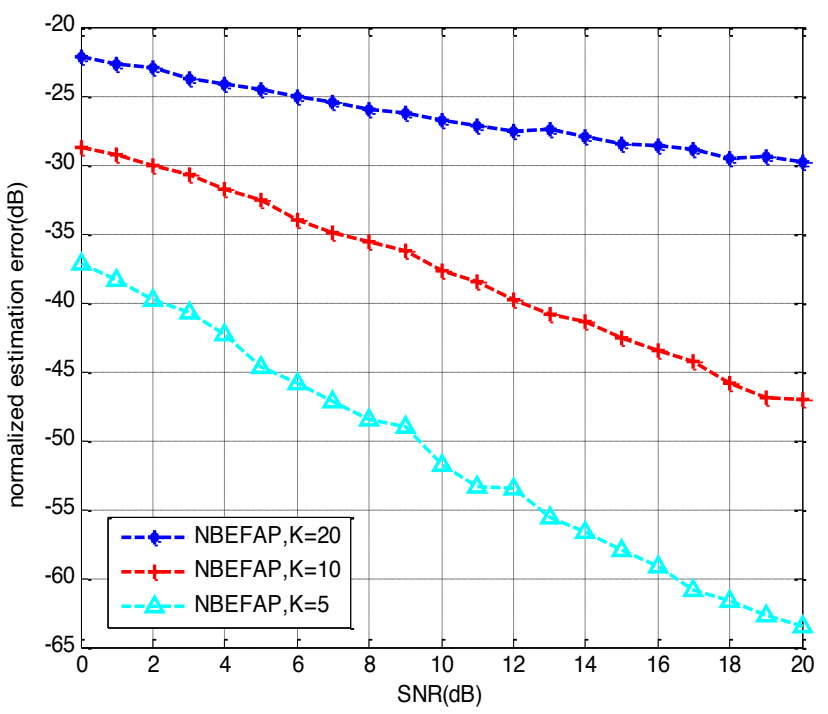

Fig. 3. MSE performance with different sparsity level, $L=20$ 
The estimation MSE performance comparison between the conventional NBEAP and the proposed NBEFAP scheme is given in Fig. 4. In NBEAP, 60 percent of the receiving antennas are randomly chosen. From the simulation result we get the following conclusion. Using the same pilot length $L=30$, the NBEFAP scheme gets better MSE performance than the NBEAP scheme. The NBEFAP scheme still has better estimation MSE performance even when its pilot length $(L=30)$ is shorter than that of the NBEAP scheme $(L=41)$. This is because that the whole received pilot data on each antenna is exploited to estimate the CSI in NBEFAP. The estimation MSE performance of LS and LMMSE algorithm are also given in Fig. 4. It shows that the NBEFAP scheme obtains better estimation MSE performance compared with the LS and LMMSE. Assuming that the user moves at the speed of $100 \mathrm{~km} / \mathrm{h}$ and the carrier frequency is $900 \mathrm{MHz}$, the corresponding channel coherence time is about $5 \mathrm{~ms}$. The periods of pilot and information data symbol are set as $0.07 \mathrm{~ms}$. The transmission efficiency is $42.6 \%$ when the length of pilot sequence is 41 . In contrast, if the length of pilot sequence is 30 , the transmission efficiency can be increased to $58 \%$.

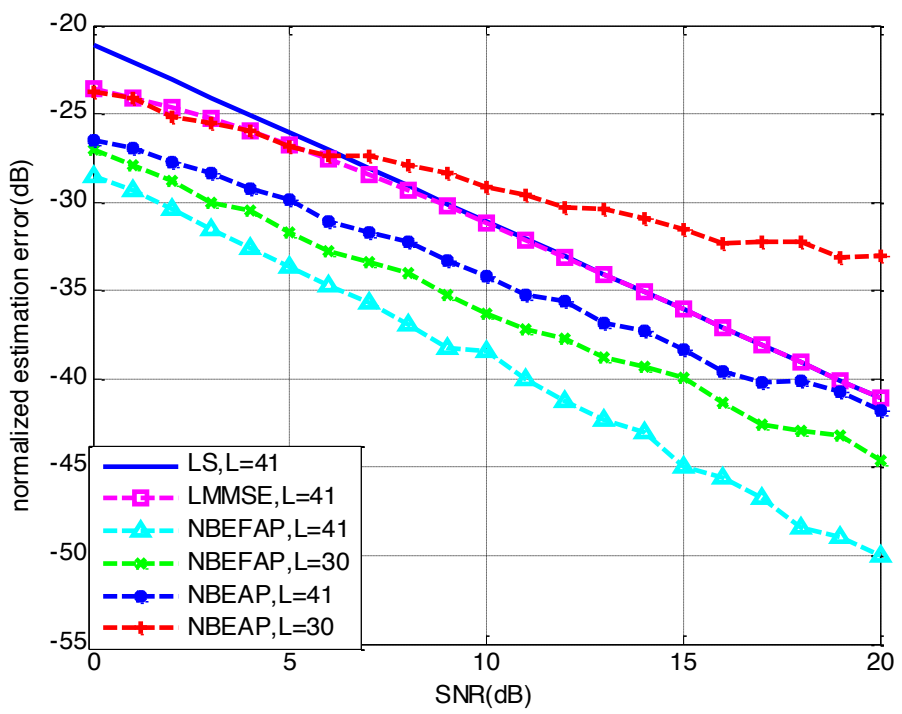

Fig. 4. Comparison of the MSE performance between NBEFAP and NBEAP

In Fig. 5, the MSE performance comparison with different numbers of antennas is given. It is observed that the MSE performance of the channel estimation is improved with the increase of the antennas. This is due to the fact that a large number of antennas provide great diversity gain.

For the case that the sparsity level of the channel changes slowly, the length of pilot sequence is adjusted adaptively according to the sparsity level. The channel estimation MSE performance is given in Fig. 6. We set the initial pilot length $L=10$, $\Delta L=5$ and $\mathrm{SNR}=5 \mathrm{~dB}$. From the simulation result we find that the estimation MSE using fixed pilot length increases with the sparsity level. In contrast, the estimation 
MSE using adaptive varying pilot length is almost constant, regardless of the sparsity level. It is important to maintain the stability of channel estimation accuracy in highorder MIMO system. Since there is no need to frequently adjust parameters such as transmission power according to the channel estimation accuracy, the complexity of the transmission is reduced.

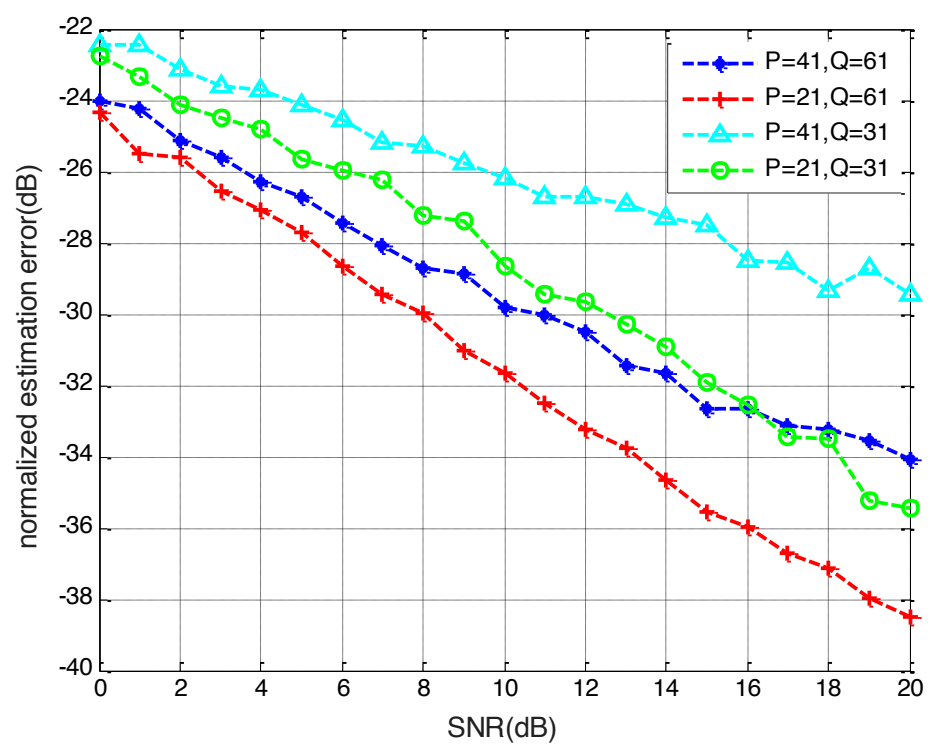

Fig. 5. Comparison of the MSE performance with different numbers of antennas

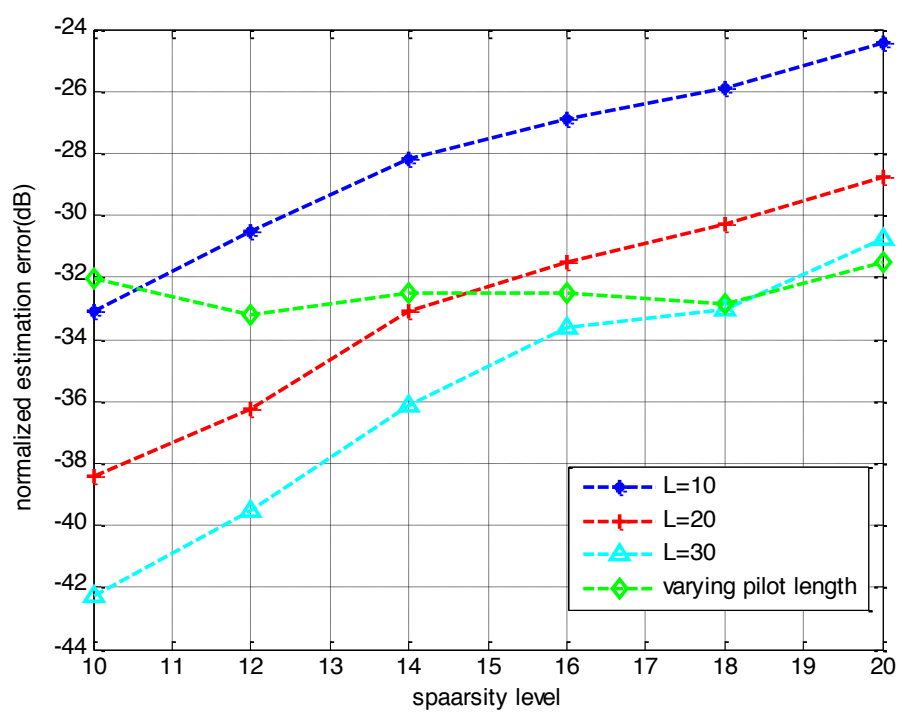

Fig. 6. Comparison of the MSE performance between fixed and varying pilot length 


\section{Conclusions}

In this paper, we propose a CS based scheme for channel estimation in high-order MIMO system. An accurate channel estimation is obtained with short pilot sequence. The key idea of the scheme is using a partition of an identity matrix as pilot matrix and estimating the CSI with CS theory. Since the pilot matrix has simple structure, the complexity of channel estimation is reduced. The received pilot signals on each receiving antenna have been fully utilized which results in an accurate estimated channel. When the sparsity level of the channel varies with time, the length of pilot sequence is adjusted dynamically to save pilot resources. Another benefit of the varying pilot length is that the channel estimation MSE performance keeps almost constant as the sparsity level increases. The simulation results validate the effectiveness of the NBEFAP scheme.

\section{$7 \quad$ Acknowledgment}

This work was supported by the national Natural Science Foundation of China under grant 61671345 and the Joint Fund of Ministry of Education of China (No.6141A02022338).

\section{References}

[1] Mehmood.Y., Afzal, W., Ahmad, F., Younas, U., Rashid, I., Mehmood, I. (2013). Large scaled multi-user MIMO system so called massive MIMO systems for future wireless communication networks, Automation and Computing (ICAC), 2013, London, UK, pp.1-4

[2] E. G. Larsson, F. Tufvesson, O. Edfors, and T. L. Marzetta. (2014). Massive MIMO for next generation wireless systems. IEEE Commun. Mag. 52: 186-195 https://doi.org/10.1109/MCOM.2014.6736761

[3] F. Rusek et al. (2013). Scaling Up MIMO: Opportunities and Challenges with Very Large Arrays, IEEE Sig. Proc. Mag. 30: 40-60 https://doi.org/10.1109/MSP.2011.2178495

[4] D. Donoho. (2006). Compressed Sensing, IEEE Transactions on Information Theory, 52:1289-1306 https://doi.org/10.1109/TIT.2006.871582

[5] W. Ding, Y. Lu, F. Yang, et al. (2016). Spectrally Efficient CSI Acquisition for Power Line Communications: A Bayesian Compressive Sensing Perspective, IEEE Journal on Selected Areas in Communications, 34: 2022-2032 https://doi.org/10.1109/JSAC. 2016.2566140

[6] W. Ding, F. Yang, C. Pan, et al. (2014). Compressive Sensing Based Channel Estimation for OFDM Systems Under Long Delay Channels, IEEE Transactions on Broadcasting, 60: 313-321 https://doi.org/10.1109/TBC.2014.2315913

[7] G. Jie, B. Song, Y. He, et al. (2017). A Survey on Compressed Sensing in Vehicular Infotainment Systems, IEEE Communications Surveys and Tutorials, 19: 2662-2680 https://doi.org/10.1109/COMST.2017.2705027

[8] J. Kivinen, P. Suvikunnas, L. Vuokko, and P. Vainikainen. (2002). Experimental investigations of MIMO propagation channels, IEEE Int. symp. Antennas and Propagation, San Antonio, TX, USA, pp.16-21. 
[9] Z. Yan, M. Herdin, A. M. Sayeed, et al. (2007). Experimental study of MIMO channel statistics and capacity via the virtual channel representation, Tech. Rep., Univ. WinsconsinMadison, http://dune.ece.wisc.edu/pdfs/zhou meas.pdf

[10] G. Gui, N. Liu, L. Xu and F. Adachi. (2015). Low-complexity large-scale multiple-input multiple-output channel estimation using affine combination of sparse least mean square filters, IET Communications, 9:2168-2175 https://doi.org/10.1049/iet-com.2014.0979

[11] A. M. Sayeed. (2002). Deconstructing multiantenna fading channels, IEEE Transactions on, Signal Processing, 50:2563-2579 https://doi.org/10.1109/TSP.2002.803324

[12] A. M. Sayeed. (2003). A virtual representation for time- and frequency-selective correlated MIMO channels, Acoustics, Speech, and Signal Processing, 2003. Proceedings. (ICASSP '03). Hong Kong, China, 4: 648-651

[13] Ping-Heng Kuo, Kung, H.T, Pang-An Ting. (2012). Compressive sensing based channel feedback protocols for spatially-correlated massive antenna arrays, Wireless Communications and Networking Conference (WCNC), Shanghai, China, pp.492-497 https://doi.org/10.1109/WCNC.2012.6214417

[14] S. Nguyen and A. Ghrayeb. (2013). Compressive sensing-based channel estimation for massive multiuser MIMO systems, IEEE Wireless Communications and Networking Conference (WCNC), Shanghai, China, pp.2890-2895.

[15] C. Rusu, R. Mendez-Rial, N. Gonzalez-Prelcic and R. W. Heath. (2015). Adaptive One-Bit Compressive Sensing with Application to Low-Precision Receivers at mmWave, IEEE Global Communications Conference (GLOBECOM), San Diego, CA, pp. 1-6. https://doi.org/10.1109/GLOCOM.2015.7417853

[16] R. Méndez-Rial, C. Rusu, A. Alkhateeb, N. González-Prelcic and R. W. Heath. (2015). Channel estimation and hybrid combining for mmWave: Phase shifters or switches?, Information Theory and Applications Workshop (ITA), San Diego, CA, pp. 90-97. https://doi.org/10.1109/ITA.2015.7308971

[17] Bajwa, W.U.; Sayeed, A.; Nowak, R. (2008). Compressed sensing of wireless channels in time, frequency, and space, 42nd Asilomar Conference. Signals, Systems and Computers, Pacific Grove, CA, USA, pp.2048-2052

[18] E. J. Candes and T. Tao. (2005). Decoding by linear programming, IEEE Trans. Inf. Theory, 51: 4203-4215 https://doi.org/10.1109/TIT.2005.858979

[19] M. F. Duarte and Y. C. Eldar. (2011). Structured compressed sensing: From theory to applications, IEEE Trans. Signal Processing, 59:4053-4085 https://doi.org/10.1109/TSP.20 $\underline{11.2161982}$

\section{Authors}

Zhiguo Lv received the B.S. degree in applied electronic technology from Henan Normal University, Xinxiang, China, in 2000, the M.S. degree in communications engineering from Guilin University of Electronic Technology, Guilin, China, in 2008. Since 2008, he has been on the faculty of Luoyang Institute of Science and Technology. He is currently working toward the Ph.D. degree at Xidian University. His research interests include MIMO communication and compressive sensing. (E-mail: lzg96w1@163.com)

Ying Li received the B.S. degree in telecommunication engineering and the Ph.D. degree in communication and information systems from Xidian University, Xi'an, China, in 1995 and 2005, respectively. From 2011 to 2012, she was with the Universi- 
ty of California, Davis, USA, as a Visiting Scholar. She is currently a Professor with Xidian University. Her current research interests are on design and analysis for wireless systems, including channel coding, wireless network communications, interference processing, and MIMO techniques.

Article submitted 10 January 2018. Resubmitted 12 March and 16 March 2018. Final acceptance 06 April 2018. Final version published as submitted by the authors. 